\title{
Anti-IL-6 receptor antibody does not ameliorate radiation pneumonia in mice
}

\author{
TOSHIYUKI OGATA ${ }^{1}$, HIDEYA YAMAZAKI ${ }^{4}$, TERUKI TESHIMA ${ }^{2}$, TAKAHIRO TSUCHIYA $^{2}$, \\ NORIHIRO NISHIMOTO ${ }^{5}$ and NARIAKI MATSUURA ${ }^{3}$
}

\author{
Departments of ${ }^{1}$ Radiation Oncology, ${ }^{2}$ Medical Physics and Engineering and ${ }^{3}$ Functional Diagnostic Science, \\ Osaka University Graduate School of Medicine, Suita, Osaka 565-0871; ${ }^{4}$ Department of Radiology, \\ Graduate School of Medical Science, Kyoto Prefectural University of Medicine, \\ Kawaramachi Hirokoji, Kamigyo-ku, Kyoto 602-856; ${ }^{5}$ Laboratory of Immune Regulation, \\ Wakayama Medical University, Ibaraki, Osaka 567-0085, Japan
}

Received March 20, 2012; Accepted May 9, 2012

DOI: $10.3892 /$ etm.2012.582

\begin{abstract}
We previously showed that early administration of monoclonal anti-interleukin-6 receptor antibody (IL-6RA) does not prevent radiation-induced lung injury in mice. The purpose of this study was to investigate whether a higher dose and longer course of IL-6RA treatment was effective in ameliorating radiation pneumonia. $\mathrm{C} 57 \mathrm{Bl} / 6 \mathrm{~J}$ mice received thoracic irradiation of $12 \mathrm{~Gy}$, and were intraperitoneally injected with the IL-6RA, namely MR16-1, or with control rat IgG 4 times, once immediately following exposure and then weekly from 1 to 3 weeks after irradiation. Enzyme-linked immunosorbent assays were used to analyze the plasma levels of IL-6 and serum amyloid A (SAA). Lung injury was assessed by histological staining with haematoxylin and eosin $(\mathrm{H} \& \mathrm{E})$ and by measuring wet lung weight. We observed marked upregulation of IL-6 in IL-6RA-treated mice compared to the IgG-treated control group, whereas IL-6RA did not increase the production of SAA in the group receiving irradiation. However, radiation pneumonia, as evaluated by H\&E staining and lung weight showed no differences between the IL-6RAtreated mice and the controls. Long-term treatment with high-dose IL-6RA does not ameliorate radiation pneumonia.
\end{abstract}

\section{Introduction}

Radiation pneumonia, an interstitial pulmonary inflammation, and subsequent radiation lung fibrosis are significant dose-limiting complications, and may threaten the quality of life of patients receiving radiation to the thorax. At the cellular and tissue levels, radiation pneumonia presents as an edema

Correspondence to: Dr Toshiyuki Ogata, Department of Radiation Oncology, Osaka University Graduate School of Medicine, 2-2 Yamadaoka, Suita, Osaka 565-0871, Japan

E-mail: ogata@radonc.med.osaka-u.ac.jp

Key words: interleukin-6, radiation pneumonia, cytokine of the interstitial space, infiltration of inflammatory cells and thickening of the alveolar septa. Although the molecular mechanism underlying radiation pneumonia is complex and obscure, pro-inflammatory cytokines, chemokines and cell adhesion molecules are likely to be implicated $(1,2)$. Many researchers have shown that cytokines play important roles in the pathogenesis of radiation pneumonia $(3,4)$. Interleukin-6 (IL-6), which was originally identified as a B-cell differentiation factor (5), is now known to be a multifunctional cytokine that regulates acute phase response, immune response and inflammation (6,7). IL-6 is produced by a variety of cells such as T cells, B cells, monocytes, macrophages, fibroblasts, endothelial cells and several tumor cells (8). Clinical as well as experimental findings have suggested the involvement of IL-6 as a pro-inflammatory cytokine in radiation pneumonia (9-12). Indeed, IL- 6 production has been found to be elevated in both humans and animals with radiation pneumonia. Therefore, we previously investigated whether monoclonal anti-IL-6 receptor antibody (IL-6RA) treatment could provide a new pharmacological intervention strategy for ameliorating radiation-induced lung injury (13). However, early intervention using IL-6RA was not able to prevent radiation-induced lung injury. One possible explanation for this finding is that long-term continuous administration of IL-6RA may be necessary to reduce lung toxicity. Radiation-induced lung injury is a chronic phenomenon mediated by a variety of cells such as inflammatory cells responding to the release or activation of downstream cytokines, growth factors or chemokines. In this study, we examined the effect of a higher dose and longer course of IL-6RA treatment on radiation pneumonia in mice following lethal whole thorax irradiation.

\section{Materials and methods}

Mice and irradiation. Eight-week-old specific pathogen-free female C57Bl/6J mice were obtained from Charles River Laboratories Japan, Inc. (Yokohama, Japan) a week before testing. The mice were maintained and the study protocol was established according to guidelines of the Institutional Animal Care and Use Committee at Osaka University Graduate School 
of Medicine. The mice were anesthetized by an intraperitoneal (i.p.) injection of pentobarbital $(40 \mathrm{mg} / \mathrm{kg})$ immediately before irradiation. The whole thorax was irradiated with $4 \mathrm{MV}$ $\mathrm{X}$-rays from a linear accelerator (EXL-6SP; Varian Medical Systems, Palo Alto, CA, USA) at a dose of 12 Gy delivered at a dose rate of $\sim 1.8 \mathrm{~Gy} / \mathrm{min}$ with a 1.0 -cm bolus material on the surface. This dose is estimated to be an approximately lethal dose (LD) 50/360 (the lethal dose required to kill $50 \%$ of the C57Bl/6J mice within 360 days) (14). The field was $2.5 \mathrm{~cm}$ in length in the cephalocaudal direction. Mice were randomly assigned to one of four treatment groups ( $\mathrm{n}=6 /$ group): radiation and IL-6RA (IL-6RA 12 Gy), radiation and control (IgG $12 \mathrm{~Gy}$ ), sham radiation and IL-6RA (IL-6RA $0 \mathrm{~Gy}$ ), and sham radiation and control (IgG $0 \mathrm{~Gy}$ ). Mice were sacrificed 30 days after irradiation.

IL-6RA (MR16-1) injection. Basic characteristics of the rat antimouse IL-6 receptor monoclonal antibody, MR16-1 (Chugai Pharmaceutical, Co., Ltd., Tokyo, Japan), have been described in a previously published study (15). Mice were first injected i.p. with $2 \mathrm{mg}$ of MR16-1, or control rat $\mathrm{IgG}$ antibody (ICN Biomedicals, Inc., Irvine, CA, USA) immediately following irradiation. Subsequent weekly injections of IgG control or $0.5 \mathrm{mg}$ of antibody were administered from 1 to 3 weeks after irradiation.

Analysis of circulating IL-6 and SAA. Blood samples were collected via cardiac puncture at the time of euthanasia and plasma was obtained by microcentrifugation at $4,000 \mathrm{x} \mathrm{g}$ for 5 min. Plasma concentrations of IL-6 (Pierce Biotechnology, Inc., Rockford, IL, USA) and serum amyloid A (SAA) (Immunology Consultants Laboratory, Inc., Newberg, OR, USA) were measured using commercial enzyme-linked immunosorbent assay (ELISA) kits according to the manufacturer's instructions.

Histological analysis. For histological examination, lung tissue was fixed in $10 \%$ neutral-buffered formalin, embedded in paraffin wax, sectioned $(5-\mu \mathrm{m})$ and stained with haematoxylin and eosin (H\&E). Following H\&E staining, we evaluated edema of the interstitial space, infiltration of inflammatory cells, thickening of the alveolar septa and vessel thrombosis.

Statistical analysis. Comparison between pairs of groups was performed using the non-parametric Mann-Whitney U test due to the small sample number. A P-value of $<0.05$ was considered to indicate statistical significance.

\section{Results}

Body weight as an indicator of the systemic toxicity of radiation pneumonia is presented graphically in Fig. 1; no differences in body weight were observed among the 4 groups.

Mouse plasma levels of IL-6 and SAA, one of the major acute-phase proteins of inflammation and tissue damage in mammals, were determined using specific ELISAs. The IL-6 levels in the IgG 0 Gy, IL-6RA 0 Gy, IgG 12 Gy and IL-6RA 12 Gy groups were 4.4 $\pm 1.2,73.9 \pm 63.8,15.6 \pm 2.7$ and $77.0 \pm 41.8 \mathrm{pg} / \mathrm{ml}$, respectively (Fig. 2A). A significant increase was observed in the IL-6 level in the IL-6RA 12 Gy group

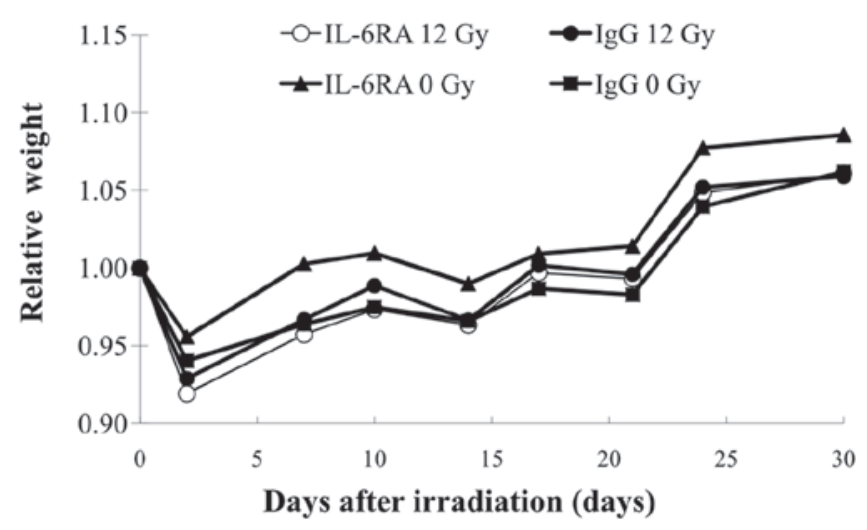

Figure 1. Effect of IL-6RA-treatment on body weight. Data are presented as relative weight using the weight at Day 0 after irradiation for normalization.
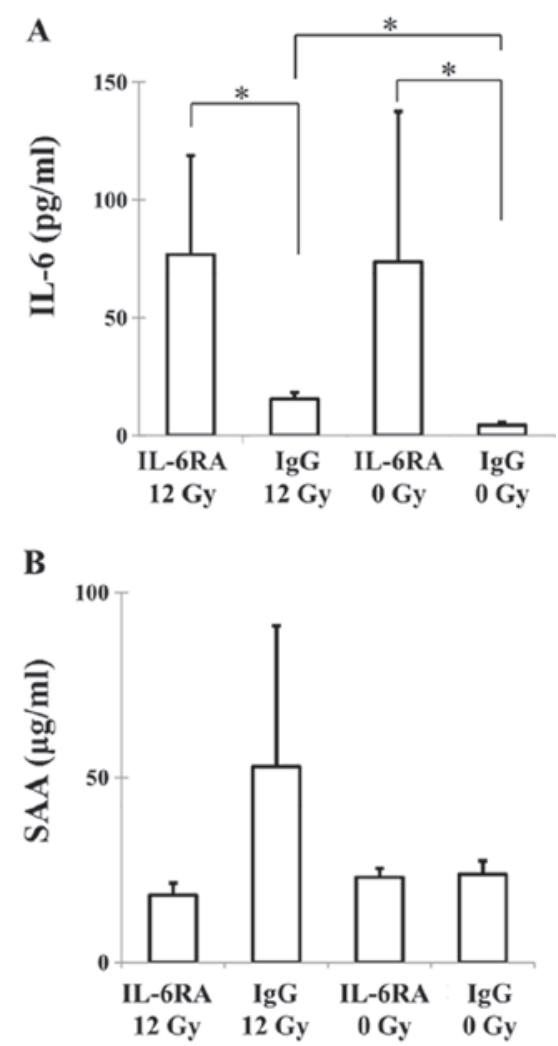

Figure 2. Effects of IL-6RA-treatment on (A) IL-6 and (B) SAA production 30 days after irradiation. Each bar represents the mean \pm SD ( $n=6 /$ group). *Statistical significance.

compared to the IgG 12 Gy group. The mice in the IL-6RA 12 Gy group also had significantly higher levels than the $\operatorname{IgG}$ 0 Gy group. A marked increase in the level of IL- 6 was found in the $\operatorname{IgG} 12 \mathrm{~Gy}$ group compared to the $\operatorname{IgG} 0 \mathrm{~Gy}$ group. The SAA level in the IgG 0 Gy, IL-6RA 0 Gy, IgG 12 Gy and IL-6RA 12 Gy groups were 23.9 $33.6,23.1 \pm 2.4,52.9 \pm 38.1$ and $18.2 \pm 3.3 \mu \mathrm{g} / \mathrm{ml}$, respectively (Fig. 2B). The mice in the IgG 12 Gy group exhibited higher SAA protein levels, but this was not statistically significant, compared to the other groups (Fig. 2B). The IL-6RA 12 Gy group showed low SAA protein levels in plasma. No significant difference was found in the SAA level between the IL-6RA 0 Gy and IgG 0 Gy groups. 


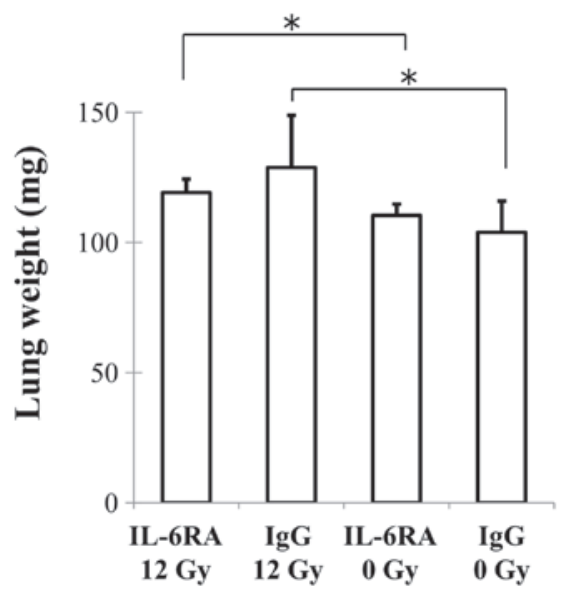

Figure 3. Effect of IL-6RA treatment on radiation-induced lung injury indices 30 days following irradiation. Wet lung weight following $12 \mathrm{~Gy}$ of single irradiation to the whole thorax. Data obtained from 6 animals in each group are presented as means \pm SD. *Statistical significance.
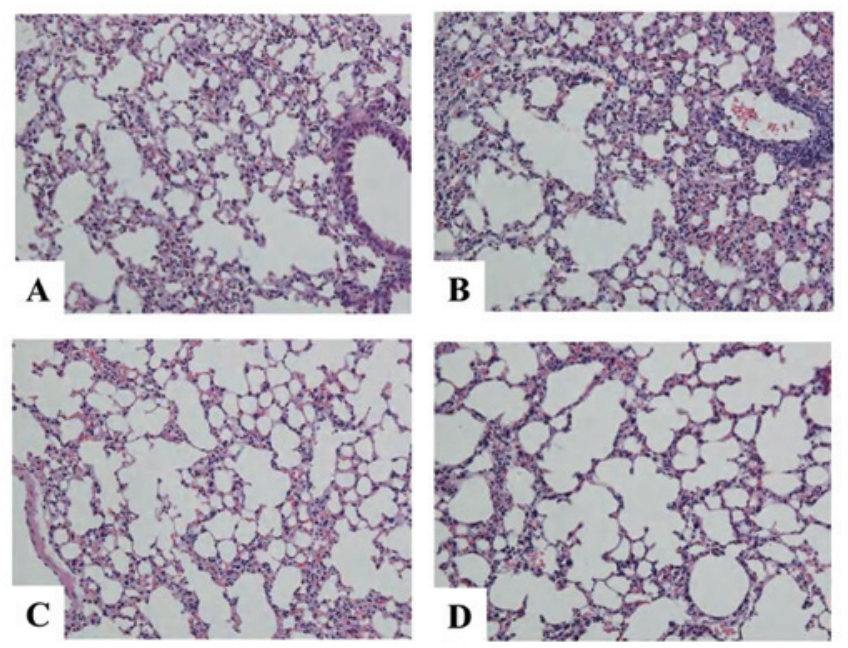

Figure 4. Histological analyses using H\&E staining for irradiated or nonirradiated mice lung tissue 30 days after irradiation. (A) IL-6RA 12 Gy, (B) IgG 12 Gy, (C) IL-6RA 0 Gy and (D) IgG 0 Gy.

Wet lung weight was determined as a measure of pulmonary edema and consolidation. There were no differences in the lung weight of the IgG 12 Gy and IL-6RA 12 Gy groups (Fig. 3).

The H\&E-stained lungs of the mice in both the IgG 12 Gy and IL-6RA 12 Gy groups showed an increased acute inflammatory infiltration in the interstitium (Fig. 4). However, no significant difference was found between the IgG 12 Gy and IL-6RA 12 Gy groups. No damage to the lung structure was observed in the IgG 0 Gy or IL-6RA 0 Gy groups.

\section{Discussion}

Radiation pneumonia and subsequent radiation lung fibrosis are major dose-limiting complications for patients undergoing thoracic radiotherapy. Recent research findings support the existence of a mechanism of cellular interaction between lung parenchymal cells and circulating immune cells, which is mediated by a variety of pro-inflammatory cytokines, chemokines, adhesion molecules and pro-fibrotic cytokines $(1,2)$.
Since IL-6 is a pleiotropic cytokine that plays essential roles in the regulation of the immune response and inflammation, IL-6 receptor monoclonal antibody treatment has been identified as a promising treatment for rheumatoid arthritis, juvenile idiopathic arthritis, Castleman's disease and Crohn's disease (16-19). IL-6 has also been implicated in the pathogenesis of radiation pneumonia (9-12) and is synthesized by type II pneumocytes, alveolar macrophages, $\mathrm{T}$ lymphocytes and lung fibroblasts (3). We therefore hypothesized that blockage of the IL-6 signaling pathways could offer an attractive therapeutic target for the amelioration of radiation-induced lung injury. However, early IL-6RA treatment was not able to mitigate radiation-induced lung injury (13).

Rübe et al (12) showed that radiation-induced release of IL-6 in the bronchiolar epithelium of $\mathrm{C} 57 \mathrm{Bl} / 6 \mathrm{~J}$ mice could be detected a few hours and several weeks after irradiation. Anscher et al (20) reported that long-term administration of the smallmolecule inhibitor of TGF- $\beta$ was more effective in reducing radiation-induced lung toxicity than short-term administration. Rabbani et al (21) demonstrated that prolonged administration of the novel catalytic anti-oxidant, AEOL 10150, after irradiation protects against radiation-induced lung injury. However, treatment with AEOL 10150 before and for a short time after irradiation had no significant benefits. Therefore, we hypothesized that long-term continuous administration of IL-6RA might be necessary to reduce lung toxicity. In this study, we used a higher dose and longer course (2 mg of MR16-1 initially, followed by 3 doses of $0.5 \mathrm{mg}$ MR16-1, weekly for 3 weeks) of IL-6RA treatment than we used in our previous study ( 2 doses of $0.2 \mathrm{mg}$ MR16-1, weekly) (13). In addition, we used the $\mathrm{C} 57 \mathrm{Bl} / 6$ strain of mice, which more easily develops radiationinduced lung injury than the Balb/c mice used in the previous study. Usage of a different mice strain or irradiation dose may have resulted in changes in the results from our previous study.

In our previous study, we were not able to administer IL-6RA more than twice, since we were concerned that repeated treatment with a rat antibody would result in the production of mouse anti-rat antibodies. Recently, Tomiyama-Hanayama et al (22) examined the effect of IL-6RA concentration, by using the treatment regimen that we used in this study, on renal injury in apolipoprotein E-deficient mice and confirmed the safety of an intensive dose.

We found a significant increase in the IL-6 levels in the radiation and IL-6RA treatment group compared to the radiation only group. Nishimoto et al (23) reported that serum IL-6 markedly increased after IL-6RA administration in both rheumatoid arthritis and Castleman's disease through inhibition of IL-6R-mediated consumption of IL-6. Despite the increase in serum IL-6 levels, IL-6RA treatment has been shown to dramatically ameliorate inflammatory manifestations and to normalize the levels of acute phase proteins such as C-reactive protein in rheumatoid arthritis and Castleman's disease. Since one possible explanation for the increase in serum IL-6 following IL-6RA treatment is that IL-6RA may inhibit the clearance of IL-6 from serum, the measurement of serum IL-6 levels only may be a limitation in evaluating radiation pneumonia. Consistent with this report, our data revealed that IL-6RA treatment maintained the same SAA protein level as in the IgG 0 Gy group. Acute phase protein SAA is known as a sensitive systemic marker of inflammation and tissue damage (24). 
Furthermore, IL-6, acting synergistically with tumor necrosis factor or IL-1, plays an important role in the induction of the SAA gene and IL-6RA inhibits this synergistic effect of IL-6 on SAA production (25). Since SAA did not increase in the IL-6RA-treated mice receiving irradiation in this study, IL-6 action may be inhibited. We previously observed that IL-6RA treatment suppressed the radiation-induced increase in IL-6 as compared with the $\mathrm{IgG}$ control group 50 days after irradiation (13). Such a discrepancy may be due to differences in the protocol of antibody administration and time of assessment.

Our findings suggest that elevation of IL- 6 may not be involved to a great extent in the mechanism behind the development of radiation pneumonia, but instead reflects the inflammatory state of the lung due to the development of radiation pneumonia. Measurement of plasma IL-6, as an acute phase inflammatory cytokine, may therefore indicate the severity of inflammatory state of the radiation-induced lung injury, although Rübe et al (26) reported that IL-6 levels do not provide a predictive risk assessment for radiation pneumonia in patients irradiated for non-small cell lung cancer. The utility of IL-6 measurement should be validated in future studies, since IL- 6 also increases in patients with pulmonary diseases such as infectious pneumonia, interstitial pneumonia and chronic obstructive pulmonary disease (27).

Limitations of our study included the lack of evaluation of data over long periods of time and the relatively small number of mice used. We evaluated radiation-induced lung injury in only acute interstitial inflammation (30 days) as IL-6 has been implicated in the pathogenesis of radiation pneumonia. Saito-Fujita T et al (28) demonstrated that IL-6-knockout mice exhibited attenuated radiation-induced lung fibrosis. Additional research is required to determine the optimal timing, antibody dose and duration for therapy using this approach for the prevention of lung injury after radiation therapy.

In conclusion, the findings demonstrated that intervention using IL-6RA does not ameliorate radiation pneumonia. Therefore, more detailed studies are required to identify the possible strategies for the inhibition of cytokine signaling as a method for mitigating lung injury caused by radiation therapy.

\section{Acknowledgements}

The authors are indebted to Kumie Hirai, Kazumasa Minami and Masaru Isono for the excellent technical support.

\section{References}

1. Tsoutsou PG and Koukourakis MI: Radiation pneumonitis and fibrosis: mechanisms underlying its pathogenesis and implications for future research. Int J Radiat Oncol Biol Phys 66: 1281-1293, 2006.

2. Brush J, Lipnick SL, Phillips T, Sitko J, McDonald JT and McBride WH: Molecular mechanisms of late normal tissue injury. Semin Radiat Oncol 17: 121-130, 2007.

3. Rubin P, Johnston CJ, Williams JP, McDonald S and Finkelstein JN: A perpetual cascade of cytokines postirradiation leads to pulmonary fibrosis. Int J Radiat Oncol Biol Phys 33: 99-109, 1995

4. Chen Y, Williams J, Ding I, et al: Radiation pneumonitis and early circulatory cytokine markers. Semin Radiat Oncol 12 : S26-S33, 2002.

5. Hirano T, Yasukawa K, Harada H, et al: Complementary DNA for a novel human interleukin (BSF-2) that induces B lymphocytes to produce immunoglobulin. Nature 324: 73-76, 1986.
6. Nishimoto $\mathrm{N}$ and Kishimoto T: Inhibition of IL-6 for the treatment of inflammatory diseases. Curr Opin Pharmacol 4: 386-391, 2004.

7. Nishimoto $\mathrm{N}$ and Kishimoto T: Interleukin-6: from bench to bedside. Nat Clin Pract Rheumatol 2: 619-626, 2006.

8. Kishimoto T, Akira S, Narazaki M and Taga T: Interleukin-6 family of cytokines and gp130. Blood 86: 1243-1254, 1995.

9. Chen Y, Rubin P, Williams J, Hernady E, Smudzin T and Okunieff P: Circulating IL-6 as a predictor of radiation pneumonitis. Int J Radiat Oncol Biol Phys 49: 641-648, 2001.

10. Chen Y, Hyrien O, Williams J, Okunieff P, Smudzin T and Rubin P: Interleukin (IL)-1A and IL-6: applications to the predictive diagnostic testing of radiation pneumonitis. Int J Radiat Oncol Biol Phys 62: 260-266, 2005.

11. Rübe CE, Wilfert F, Palm J, et al: Irradiation induces a biphasic expression of pro-inflammatory cytokines in the lung. Strahlenther Onkol 180: 442-448, 2004.

12. Rübe CE, Uthe D, Wilfert F, et al: The bronchiolar epithelium as a prominent source of pro-inflammatory cytokines after lung irradiation. Int J Radiat Oncol Biol Phys 61: 1482-1492, 2005.

13. Ogata T, Yamazaki H, Teshima T, et al: Early administration of IL-6RA does not prevent radiation-induced lung injury in mice. Radiat Oncol 5: 26, 2010.

14. Chiang CS, Liu WC, Jung SM, et al: Compartmental responses after thoracic irradiation of mice: strain differences. Int J Radiat Oncol Biol Phys 62: 862-871, 2005.

15. Okazaki M, Yamada Y, Nishimoto N, Yoshizaki K and Mihara M: Characterization of anti-mouse interleukin- 6 receptor antibody. Immunol Lett 84: 231-240, 2002.

16. Nishimoto N, Yoshizaki K, Miyasaka N, et al: Treatment of rheumatoid arthritis with humanized anti-interleukin-6 receptor antibody: a multicenter, double-blind, placebo-controlled trial. Arthritis Rheum 50: 1761-1769, 2004.

17. Yokota S, Miyamae T, Imagawa T, et al: Therapeutic efficacy of humanized recombinant anti-interleukin- 6 receptor antibody in children with systemic-onset juvenile idiopathic arthritis. Arthritis Rheum 52: 818-825, 2005.

18. Nishimoto N, Kanakura Y, Aozasa K, et al: Humanized antiinterleukin-6 receptor antibody treatment of multicentric Castleman disease. Blood 106: 2627-2632, 2005.

19. Ito H, Takazoe M, Fukuda Y, et al: A pilot randomized trial of a human anti-interleukin-6 receptor monoclonal antibody in active Crohn's disease. Gastroenterology 126: 989-996, 2004.

20. Anscher MS, Thrasher B, Zgonjanin L, et al: Small molecular inhibitor of transforming growth factor-beta protects against development of radiation-induced lung injury. Int J Radiat Oncol Biol Phys 71: 829-837, 2008.

21. Rabbani ZN, Batinic-Haberle I, Anscher MS, et al: Long-term administration of a small molecular weight catalytic metalloporphyrin antioxidant, AEOL 10150, protects lungs from radiation-induced injury. Int J Radiat Oncol Biol Phys 67: 573-580, 2007.

22. Tomiyama-Hanayama M, Rakugi H, Kohara M, et al: Effect of interleukin-6 receptor blockage on renal injury in apolipoprotein E-deficient mice. Am J Physiol Renal Physiol 297: F679-F684, 2009.

23. Nishimoto N, Terao K, Mima T, Nakahara H, Takagi N and Kakehi T: Mechanisms and pathologic significances in increase in serum interleukin-6 (IL-6) and soluble IL-6 receptor after administration of an anti-IL-6 receptor antibody, tocilizumab, in patients with rheumatoid arthritis and Castleman disease. Blood 112: 3959-3964, 2008.

24. Gabay C and Kushner I: Acute-phase proteins and other systemic responses to inflammation. N Engl J Med 340: 448-454, 1999.

25. Hagihara K, Nishikawa T, Isobe T, Song J, Sugamata Y and Yoshizaki K: IL-6 plays a critical role in the synergistic induction of human serum amyloid A (SAA) gene when stimulated with proinflammatory cytokines as analyzed with an SAA isoform real-time quantitative RT-PCR assay system. Biochem Biophys Res Commun 314: 363-369, 2004.

26. Rübe CE, Palm J, Erren M, et al: Cytokine plasma levels: reliable predictors for radiation pneumonitis? PLoS One 3: e2898, 2008.

27. Barthelemy-Brichant N, Bosquée L, Cataldo D, et al: Increased IL- 6 and TGF- $\beta 1$ concentrations in bronchoalveolar lavage fluid associated with thoracic radiotherapy. Int J Radiat Oncol Biol Phys 58: 758-767, 2004.

28. Saito-Fujita T, Iwakawa M, Nakamura E, et al: Attenuated lung fibrosis in interleukin 6 knock-out mice after C-ion irradiation to lung. J Radiat Res 52: 270-277, 2011. 